\title{
Neurobiological substrates of cognitive rigidity and autonomic inflexibility in generalized anxiety disorder
}

\author{
Cristina Ottaviani ${ }^{\mathrm{a}, *}$, David R. Watson ${ }^{\mathrm{b}}$, Frances Meeten ${ }^{\mathrm{b}, \mathrm{c}}$, Elena Makovac ${ }^{\mathrm{a}, \mathrm{b}}$, \\ Sarah N. Garfinkel ${ }^{b, d}$, Hugo D. Critchley ${ }^{\mathrm{b}, \mathrm{d}}$ \\ a Santa Lucia Foundation, Rome, Italy \\ b Department of Psychiatry, Brighton and Sussex Medical School, University of Sussex, Brighton, UK \\ ${ }^{\mathrm{c}}$ Kings College London, London, UK \\ d Sackler Centre for Consciousness Science, University of Sussex, Brighton, UK
}

\section{A R T I C L E I N F O}

\section{Article history:}

Received 12 November 2015

Received in revised form 20 June 2016

Accepted 22 June 2016

Available online 23 June 2016

\section{Keywords:}

Generalized anxiety disorder

Perseverative cognition

Heart rate variability

FMRI

Attention

\begin{abstract}
A B S T R A C T
Generalized anxiety disorder (GAD) is characterized by difficulties in inhibiting both perseverative thoughts (worry and rumination) and autonomic arousal. We investigated the neurobiological substrates of such abnormal inhibitory processes, hypothesizing aberrant functional coupling within 'default mode' (DMN) and autonomic brain networks. Functional imaging and heart rate variability (HRV) data were acquired from GAD patients and controls during performance of three tracking tasks interspersed with a perseverative cognition (PC) induction. After detection of infrequent target stimuli, activity within putative DMN hubs was suppressed, consistent with a redirection of attentional resources from internal to external focus. This magnitude of activity change was attenuated in patients and individuals with higher trait PC, but was predicted by individual differences in HRV. Following the induction of PC in controls, this pattern of neural reactivity became closer to that of GAD patients. Results support, at a neural level, the association between cognitive inflexibility and autonomic rigidity.
\end{abstract}

(c) 2016 Elsevier B.V. All rights reserved.

\section{Introduction}

In their "autonomic flexibility-neurovisceral integration model of anxiety", Friedman and Thayer (1998) view anxiety as a systemic inflexibility, grounded in poor inhibition. Indeed, anxious individuals show a reduced capacity to inhibit cognitive (worry), behavioural (avoidance), and accompanying physiological (reduced vagal tone) manifestations. According to this model, vagal tone, indexed by heart rate variability (HRV) may serve as a measure of the integrity of brain networks that regulate inhibition through central-autonomic nervous control in the service of efficient functioning (Friedman, 2007).

An earlier laboratory study applied this model to perseverative cognition (i.e., worry and rumination) in healthy individuals, hypothesizing a link between cognitive inflexibility and autonomic rigidity expressed as reduced HRV (Ottaviani, Shapiro, \& Couyoumdjian, 2013). The rationale for collapsing worrisome and ruminative thoughts into a single phenomenal category is

\footnotetext{
* Corresponding author at: Neuroimaging Laboratory, Santa Lucia Foundation, Via Ardeatina 306, 00142 Rome, Italy.

E-mail address: cristina.ottaviani@uniroma1.it (C. Ottaviani).
}

corroborated by studies showing no differences between these two processes on their impact on appraisals and strategies (e.g., Segerstrom, Tsao, Alden, \& Craske, 2000; Watkins, Moulds, \& Mackintosh, 2005) and by the incremental benefits of using perseverative cognition as a transdiagnostic symptom (McEvoy, Watson, Watkins, \& Nathan, 2013; Spinhoven, Drost, van Hemert, \& Penninx, 2015). In Ottaviani et al. (2013), participants performed a lowdemanding tracking task before and after a perseverative cognition induction in which they were asked to recall a past or future personally relevant negative event. The task required participants to keep the cursor inside a white circle in motion on a black screen and press the left mouse button as fast as possible each time the circle turned red. Reaction times were used as an objective index of cognitive rigidity. At different time intervals, probes interrupted the task to inquire about subjects' moods and thoughts. Whenever subjects reported worrying about a future event, or ruminating about a past stressful event, they were asked how much they experienced the thought as intrusive and how much they were trying to suppress it (subjective measures of cognitive rigidity). HRV was monitored throughout the task as an index of autonomic flexibility. Worry and rumination were expressed along attentional (slower reaction times), cognitive (efforts to inhibit intrusive thoughts), affective (mood worsening), and autonomic (lower HRV) dimensions. In line 
with Friedman and Thayer's model (1998), the cognitive inflexibility that characterized perseverative cognition was mirrored, at a physiological level, by autonomic rigidity. Two 24-h ambulatory studies replicate these findings in healthy (Ottaviani, Medea et al., 2015) and depressed participants (Ottaviani, Shahabi et al., 2015).

The present study aimed to extend these results by defining neural substrates of the cognitive rigidity that characterizes perseverative cognition using the same task and induction procedure in an fMRI environment with simultaneous cardiac monitoring. HRV was derived to test, at a neural level, the association with autonomic rigidity. Importantly, we examined these effects in both healthy participants and patients with GAD, a clinical population that has perseverative cognition as a core symptom. The simultaneous assessment of bodily reactions and cognitive processes is particularly needed when studying generalized anxiety, where changes in autonomic nervous function, such as palpitations or sweating, can drive negative cognitions creating a vicious cycle that plays a major role in the maintenance of the disorder.

The integration of cognitive and affective neuroscience with clinical autonomic research has advanced our understanding of the neurobiology of GAD and related anxiety disorders. Anxiety is associated with aberrant (often exaggerated) functional activation of brain regions normally engaged in response to motivational salient stimuli and demanding behavioural challenges. These same brain regions are also implicated in the generation (e.g., anterior cingulate cortex), representation (e.g., insular cortex) or both (e.g., amygdala) of autonomic states of arousal (Critchley, Eccles, \& Garfinkel, 2013). These studies are informed by observations in patients with clinical perturbations in autonomic response (e.g., Critchley, Mathias, \& Dolan, 2002) and recent studies illustrating how neural responses to feedback from the heart can dynamically shape the perception and processing of threat and safety (reviewed in Garfinkel \& Critchley, 2016).

Unfortunately, no published studies examining perseverative cognition in GAD have combined neuroimaging techniques with concurrent measures of peripheral autonomic nervous activity. In fact, to our knowledge, only two published studies directly investigated the brain correlates of worry in GAD patients. One of them had a small sample size ( $\mathrm{n}=6$ patients) and the absence of a comparison group as strong limitations (Hoehn-Saric, Schlund, \& Wong, 2004). Although difficult to generalize, patients showed enhanced activation of frontal and anterior cingulate (ACC) cortices in response to worrisome sentences (Hoehn-Saric et al., 2004). The second suggested that worry induction engaged the same neural system in both GAD and healthy individuals (Paulesu et al., 2010). Activity within ACC and dorsal medial prefrontal cortex (DMPFC) was enhanced by worry inducing sentences in both GAD patients and controls but, interestingly, GAD patients also showed a sustained activation of these areas during resting state scans that followed the worrying induction phase. In line with a dimensional view of psychopathology, this persistent activation correlated with dispositional tendencies to worry assessed by the Penn State Worry Questionnaire (PSWQ; Meyer, Miller, Metzger, \& Borkovec, 1990).

Overall there is need for greater neurobiological understanding of difficulties in inhibiting perseverative thoughts and autonomic dysregulation in GAD, perceived as uncontrollable and disruptive to patients' daily life. We therefore undertook a neuroimaging study, acquiring fMRI and concomitant HRV data from GAD patients and healthy controls. Participants performed repetitions of a low-demand tracking task. This task promoted occurrence of self-generated thoughts, and the degree to which these thoughts distracted participants from the task could be measured by examining pre- to post-infrequent targets brain activation patterns.

'Spontaneous' (i.e., task-free) brain activity in functional imaging experiments is no longer simply viewed as noise or a confound to be controlled (e.g., Binder et al., 1999). This motivated our ana- lytical approach: periods of low cognitive, emotional, or sensory demand (as in our pre-target epochs), reliably enhance activity across a specific network of regions proposed to support the brain's default mode (Raichle et al., 2001). The key emergent property of DMN activation is the production of self-generated thoughts or mind wandering (Mason et al., 2007). When a task requires our attention, however, the activation of such network is suppressed (as in our post-target epochs). Deficits in DMN suppression are reported in several mental illnesses, notably anxiety disorders (Anticevic et al., 2012). This observation suggests that difficulties in switching from rest to task are linked to specific symptomatology, e.g., impaired attention and worry (Forster, Nunez Elizalde, Castle, \& Bishop, 2015). In line with this approach, instead of looking at what happens while participants perform a task, it can be more informative to compare periods of resting activity before and after infrequent target occurrence. The concept of task-rest interactions acknowledges the modulation of resting brain activation by the preceding stimulus-induced activation or vice-versa (Northoff, Qin, \& Nakao, 2010). Thus, 15 s periods immediately preceding experience sampling probes were used to demonstrate activation of DMN regions during mind wandering (Christoff, Gordon, Smallwood, Smith, \& Schooler, 2009). Post-stimulus alteration in neural activity is dissociable from stimuli-evoked neural responses (Mullinger, Mayhew, Bagshaw, Bowtell, \& Francis, 2013).

Between repetitions of the task, we included an induction of perseverative cognition to increase the likelihood of worry and rumination. From here on, the use of "self-generated thoughts" will indicate thoughts that are unrelated to the immediate external environment (i.e., the ongoing task) and are internally-driven (Andrews-Hanna, Smallwood, \& Spreng, 2014). Self-generated thoughts can take the form of perseverative cognition in GAD or in healthy individuals - after an experimental induction. The term "attentional control" will be conversely used to indicate moments in which participants' attention is on the task (i.e., externallyoriented).

At a behavioural level, we expected to replicate previous findings (Ottaviani et al., 2013) showing that reaction times are delayed and HRV is reduced following induction, due to an increase in rumination and worry. Within the brain, we hypothesized that enhanced self-referential processing (at baseline in GAD and following the perseverative cognition induction in $\mathrm{HC}$ ) is associated with increased engagement of the default mode network which in turn accounts for difficulties in focusing on the tracking task (i.e., levels of default mode network de-activation at target appearance). To inform our understanding of HRV as a putative marker for neural processes involved in maladaptive cognition (Thayer, Ahs, Fredrikson, Sollers, \& Wager, 2012), we tested if higher levels of HRV predict better ability to deactivate the default mode network during the transition from internal thoughts to task-focused behaviour.

\section{Methods and materials}

\subsection{Participants}

We recruited 40 individuals to take part in the study, which was approved by the National Research Ethics Service (NRES) for the National Health Service (NHS) with university sponsorship granted via the Brighton and Sussex Medical School Research Governance and Ethics Committee. Participants were compensated for their time. One GAD subject had to be excluded from the analyses because of missing data. The final sample was composed of 19 individuals who met diagnostic criteria for generalized anxiety disorder (GAD; 17 women, 2 men; mean age \pm S.D. $=29.6 \pm 6.9$ years) and 20 healthy controls ( 17 women, 3 men; mean age $=28.7 \pm 9.5$ years). 
Only 1 participant was non-Caucasian. Patients were recruited from public advertisement and from Access and Recovery Services clinics of Sussex Partnership NHS (Mental Health) Foundation Trust. Healthy control participants were recruited by advertisement from staff and students of the University of Sussex, and Brighton and Sussex Medical School. All participants were right-handed, native English speakers, and had normal or corrected-to-normal vision.

Exclusionary criteria were: age younger than 18 years, prior history of head injury, major medical neurological or psychiatric disorder (other than GAD and co-morbid depression for the patient group), cognitive impairment, history of substance or alcohol abuse or dependence, diagnosis of heart disease, obesity (body mass index $>30 \mathrm{~kg} / \mathrm{m}^{2}$ ), pregnancy, claustrophobia or other general MRI exclusions. The exclusion of co-morbid psychiatric disorders other than depression was due to: (a) the knowledge that the psychiatric diagnosis occurring most often in comorbidity with GAD is major depressive disorder (e.g., Beesdo, Pine, Lieb, \& Wittchen, 2010); (b) the need to have an - as much as possible - unmedicated sample; (c) the focus of the paper on poor inhibition as a key factor in anxiety and the need to exclude confounding variables in drawing our conclusions (i.e., our hypotheses were driven by previous data on autonomic and neural correlates of generalized anxiety disorder without considering the effects of other comorbid disorders).

Two GAD participants were included who use long-term medications ( 1 citalopram, 1 pregabalin) at the time of the study. All other patients and controls were medication free. All participants provided written informed consent.

\subsection{Procedure}

The Structured Clinical Interview for DSM-V (SCID) was administered by a trained postdoctoral fellow (FM) to both patient and controls to confirm/exclude the diagnosis of GAD and comorbid disorders. Participants then completed a series of online socio-demographic and dispositional traits questionnaires. Participants were subsequently familiarized with the neuroimaging environment, connected to the physiological recording equipment, and then underwent the fMRI protocol.

\subsection{Questionnaires}

All participants completed a set of questionnaires accessing socio-demographic, and lifestyle (nicotine, alcohol, and caffeine consumption, physical activity) information.

Dispositional perseverative cognition was assessed by:

1) Stress-Reactive Rumination Scale (SRRS; Robinson \& Alloy, 2003), a measure of the tendency to engage in perseverative cognition after stressful events. The scale was designed to measure rumination in a manner that is not confounded with depressive symptoms, a limitation of many other self-report perseverative cognition scales (Robinson \& Alloy, 2003). The scale has the following subscales: Negative Inferential Style, Hopelessness, and Active Problem-Solving.

2) Penn State Worry Questionnaire (PSWQ; Meyer et al., 1990), a 16-item self-report questionnaire commonly used to measure pathological worry in both clinical and non-clinical populations. Respondents report how true each statement is for them on a 5-point Likert scale ( $1=$ not at all typical of me, 5=very typical of me). The PSWQ has shown good-to-excellent internal consistency ( $\alpha s=0.86-0.93$ across clinical and college samples; Molina \& Borkovec, 1994) and good discriminant validity (i.e., discriminates GAD from other anxiety disorders; Brown, Antony, \& Barlow, 1992).
To better characterize the sample from a clinical point of view, levels of state and trait anxiety (State-Trait Anxiety Inventory, STAI; Spielberger, Gorsuch, Lushene, Vagg, \& Jacobs, 1983) and depression (Beck Depression Inventory, BDI; Beck, Steer, \& Garbin, 1988) were also assessed (see Makovac et al., 2015 for further details).

\subsection{Experimental task and design}

While in the scanner, participants performed a series of three 6-min easy visuomotor tracking tasks interspersed by four 5-min resting state periods. The first and last runs were resting state runs. There were no time intervals between subsequent runs, and the next run began as soon as the participant completed the visuoanalogue scales (VASs; see the section below) at the end of the preceding run.

The tracking task (adapted from Ottaviani et al., 2013) required participants to follow a white circle moving slowly and horizontally on the screen and to press a button as fast as possible each time the circle turned red. The duration of the red circle (target) was $100 \mathrm{~ms}$ after which the circle returned to white and the task continued. For each target, accuracy and reaction times were recorded. The circle made six transits of about a minute each. Targets were presented at random intervals: there were a total of 9 targets with a maximum of 2 targets (at least $20 \mathrm{~s}$ apart) per transit. The level of difficulty was very low, to increase the likelihood of episodes of perseverative cognition. Randomly, prior to either the second or third performance of the tracking task participants underwent a verbal induction procedure designed to engender perseverative negative cognition. Participants were randomised according to when the induction occurred to permit control of temporal order effects. The induction took the form of a recorded verbal instruction delivered to the participant as follows:

"Next I would like you to recall an episode that happened in the past year that made you feel sad, anxious, or stressed, or something that may happen in the future that worries you. Then, I would like you to think about this episode in detail, for example about its possible causes, consequences, and your feelings about it. Please keep thinking about this until the end of the next tracking task. Thank you. Please take as much time as you need to recall the episode and press the button whenever you are ready".

This type of induction has been widely used and proved to be effective in eliciting perseverative cognition in healthy and pathological subjects (Ottaviani et al., 2016 for a meta-analysis).

\subsection{Visual analogue scales (VAS)}

After each tracking task (and resting state run), to assess state levels of perseverative cognition over the preceding period, participants were asked about their thoughts, using VASs.

The participants were asked to rate on separate 100-point VASs "how much, for the duration of the task, they were": (1) focused on the task? (2) distracted by external stimuli? (3) ruminating/worrying? and (4) distracted by internal thoughts?

\subsection{Physiological data processing}

Heartbeats were monitored using MRI-compatible finger pulse oximetry (8600FO; Nonin Medical). The analog physiological signals were digitized at $1000 \mathrm{~Hz}$ using a CED 1401 analog-to-digital converter (Cambridge Electronic Design) and analysed using Spike 2 software (Cambridge Electronic Design). Each signal epoch was manually checked and corrected for artefacts. As the root mean square successive difference (RMSSD) has been demonstrated to be a reliable parameter for assessing vagus-mediated HRV from very short (down to $10 \mathrm{~s}$ ) heart rate recordings (e.g., Nussinovitch 
et al., 2011), HR and RMSSD were obtained for each task epoch using RHRV 4.0 (http://rhrv.r-forge.r-project.org). As recommended, HRV data was converted to natural logarithms (lnHRV).

\subsection{MRI acquisition and image pre-processing}

All images were acquired on a 1.5-T Siemens Magnetom Avanto scanner. Blood oxygenation level dependent (BOLD) signals were recorded using spin echo sequence, 34 slices, slice thickness $3 \mathrm{~mm}$, Inter-slice gap $0.6 \mathrm{~mm}$, voxel size $3 \times 3 \times 3 \mathrm{~mm}$, TR $2520 \mathrm{~ms}$, TE $43 \mathrm{~ms}$.

For the fMRI pre-processing the first four volumes were discarded to allow the stabilisation of longitudinal magnetization. Pre-processing was conducted using FSL (FMRIB Software Library, www.fmrib.ox.ac.uk/fsl). Motion correction was conducted using FMRIB's Linear Registration Tool (MCFLIRT; Jenkinson, Bannister, Brady, \& Smith, 2002; Jenkinson \& Smith, 2001). Slice timing correction, spatial smoothing with a Gaussian kernel of $8 \mathrm{~mm}$ (FWHM), and high pass temporal filtering (cut-off full-width $100 \mathrm{~s}$ ) to remove low-frequency drift were applied. The EPI data was then registered to the individual's high-resolution structural scan using the Boundary-Based Registration (BBR) algorithm. Registration to Montreal Neurological Institute (MNI) standard space was then implemented using FNIRT (FMRIB's non-linear registration tool) nonlinear registration.

\section{Statistical analyses}

\subsection{Questionnaire, behavioural, and HRV analyses}

All data are expressed as means $\pm S D$. Differences at $\mathrm{p} \leq 0.05$ are regarded as significant. Data analyses were performed with SPSS 22.0 for Windows (SPSS Inc., USA).

Analyses on pre-existing group differences and the effects of the induction at a behavioural (RTs), subjective (VAS), and physiological (RMSSD) level are described elsewhere, although on a slightly different sample size (Makovac et al., 2015). For clarity purposes, however, the main findings will be summarized in the Results section.

To examine the role of HRV as a marker for cognitive rigidity, Pearson correlations were performed between baseline HRV and change scores in perseverative cognition from pre- to postinduction.

\section{2. fMRI data analysis}

All 1st Level and Higher Level analyses were performed using FEAT Version 6.0, part of FSL(FMRIB's Software Library, www.fmrib. ox.ac.ul//fsl).

\subsection{First-level analyses}

BOLD response was modelled using two explanatory variables; the first represented a $15 \mathrm{~s}$ pre-target period (proxy of internallyoriented attention) and the second a $15 \mathrm{~s}$ post-target period (proxy of task-oriented attention). We opted for $15 \mathrm{~s}$ in light of past evidence showing that the mean duration of each mind-wandering episode is about $14 \mathrm{~s}$ (Klinger, 1978). Our approach is consistent with previous work that, despite the lack of knowledge as to how long participants had actually been in a particular mind-wandering state, based the analyses on the assumption that the $15 \mathrm{~s}$ prior to each target/probe would consistently fall within that mindwandering state on average (e.g., He, Becic, Lee, \& McCarley, 2011; Smallwood, Beach, Schooler, \& Handy, 2008). The appearance of infrequent targets is supposed to force participants to go back on task whereas, given the monotony of the task and the infrequency of target appearance, periods immediately prior to the latter are likely to be characterized by internally-oriented attention (e.g., Smallwood, 2011). The tracking task used in the present study has proven to be effective in eliciting episodes of mind wandering (before induction) and perseverative cognition (after induction) in healthy individuals (Ottaviani et al., 2013).

These were used for the 1st Level FEAT analysis. For each explanatory variable, the regressor was convolved with a gamma function to produce an expected BOLD response. The temporal derivative of this time-course was also included in the analytic model for each variable to capture temporal shifts. Two contrasts were tested: pre-target activation greater than post-target activa-

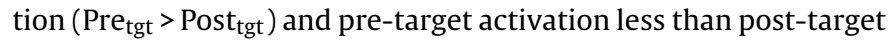
activation $\left(\right.$ Pre $_{\text {tgt }}<$ Post $_{\text {tgt }}$ ).

\subsection{Second-level analyses}

After analysing the tracking tasks before and after the induction for each subject, data were passed into a higher-level analysis, which allowed within- and between-groups comparisons. The interspersed resting-state periods go beyond the focus of the present work and will be excluded from the analyses. Higher-level analyses were carried out using FLAME (FMRIB's Local Analysis of Mixed Effects), such that group-level effects were modelled using random effects. Z-statistics images were threshold based upon clusters determined by $z>2.3$ and a (corrected) cluster significance threshold of $\mathrm{p}<0.05$. Models were created to examine the following properties:

1. Main effect of GROUP (GAD vs HC);

2. Main effect of INDUCTION (Before vs After);

3. GROUP x INDUCTION interaction.

For visualization purposes, the coefficients of parameter estimates for regions that showed significant activation differences in these contrasts were extracted and plotted.

In addition to the above analyses, to explore individual differences in cognitive functioning and brain activation, specific correlational analyses were undertaken. These tested for association between key physiological and behavioural measures and neural activity. Variables of interest were SRRS (tendency to engage in perseverative cognition) and HRV. These were modelled as explanatory variables in whole brain regression analyses. Separate analyses were performed for pre- and post-induction blocks for HRV.

Outliers were controlled for, using automatic outlier deweighting in FSL. This tool automatically detects outlier data points (for each voxel, each participant's data is considered with respect to the others to determine if it is an outlier). Outliers are then automatically de-weighted in the multi-participant statistics. All fMRI data shown were cluster-corrected for multiple comparison at $z>2.3$, $\mathrm{p}<0.05$. For visualization purposes, coefficients of parameter estimates for regions that showed significant correlations with the physiological and behavioural variables were extracted and plotted for these measures.

\section{Results}

\subsection{Descriptive results}

No group differences for any of the assessed socio-demographic and lifestyle variables emerged, therefore these were not included as covariates in the subsequent analyses (see Makovac et al., 2015). GAD participants had higher (and pathological) scores on the PSWQ $(69.1 \pm 8.2$ vs $42.6 \pm 13.4, t(38)=7.02, p<0.001)$ and the 
Table 1

Maxima of regions showing a significant $(z>2.3$, cluster corrected at $\mathrm{p}<0.05)$ main effect of Target for Pre $_{\text {tgt }}>$ Post $_{\text {tgt }}(a)$ and Post $t_{\text {tgt }}>$ Pre $_{\text {tgt }}(b)$ contrasts across groups.

\begin{tabular}{|c|c|c|c|c|c|c|}
\hline Contrast & $\mathrm{Z}$ & $\mathrm{x}$ & $\mathrm{y}$ & $\mathrm{z}$ & Hemisphere & Region of activation \\
\hline \multirow[t]{18}{*}{ (a) Pre $_{\text {tgt }}>$ Post $_{\text {tgt }}$} & 4.27 & -38 & -22 & 52 & Left & PreCentral Gyrus \\
\hline & 3.9 & 10 & -12 & 62 & Right & Juxtapositional Lobule Cortex \\
\hline & 3.44 & 12 & -16 & 72 & Right & PreCentral Gyrus \\
\hline & 3.42 & -8 & -4 & 66 & Left & Juxtapositional Lobule Cortex \\
\hline & 3.35 & -36 & -12 & 58 & Left & PreCentral Gyrus \\
\hline & 3.3 & -18 & -14 & 58 & Left & PreCentral Gyrus \\
\hline & 3.52 & -38 & -70 & 4 & Left & Lateral Occipital Cortex \\
\hline & 3.01 & -42 & -28 & 22 & Left & Parietal Operculum Cortex \\
\hline & 2.99 & -12 & -50 & 16 & Left & Precuneus \\
\hline & 2.97 & -38 & -28 & 22 & Left & Parietal Operculum Cortex \\
\hline & 2.95 & -10 & -66 & 20 & Left & Precuneus \\
\hline & 2.88 & -30 & -94 & -6 & Left & Occipital Pole \\
\hline & 3.93 & 44 & -88 & -4 & Right & Lateral Occipital Cortex \\
\hline & 3.69 & 36 & -96 & 10 & Right & Occipital Pole \\
\hline & 3.46 & 40 & -90 & 6 & Right & Occipital Pole \\
\hline & 3.31 & 32 & -84 & -2 & Right & Lateral Occipital Cortex \\
\hline & 3.13 & 42 & -62 & -8 & Right & Lateral Occipital Cortex \\
\hline & 3.04 & 44 & -68 & -4 & Right & Lateral Occipital Cortex \\
\hline \multirow[t]{6}{*}{ (b) Post $_{\text {tgt }}>$ Pre $_{\text {tgt }}$} & 4.02 & -46 & -56 & -38 & Left Cerebellum & Crus I \\
\hline & 3.89 & -40 & -66 & -44 & Left Cerebellum & Crus II \\
\hline & 3.38 & -12 & -90 & -24 & Left Cerebellum & Crus I \\
\hline & 3.17 & -34 & -82 & -28 & Left Cerebellum & Crus I \\
\hline & 3.12 & -16 & -86 & -22 & Left Cerebellum & Crus I \\
\hline & 3.1 & -20 & -80 & -22 & Left Cerebellum & Crus I \\
\hline
\end{tabular}

SRRS $(1398 \pm 263$ vs $983 \pm 238, t(38)=4.92, p<0.001)$ compared to controls. Levels of trait anxiety were significantly higher in the GAD group $(55.2 \pm 8.8$ vs $35.8 \pm 9.4, t(38)=7.01, p<0.001)$ compared to controls. According to Beck and Steer (1984) cut-offs, GAD participants reported mild-to-moderate levels of depression $(16.1 \pm 10.2)$, whereas the control group was in the no-depression range $(4.1 \pm 4.9), t(38)=4.81, p<0.001$.

\subsection{Induction effect at a behavioural, cognitive and physiological level}

After the perseverative cognition induction, all participants were less focused on task $(F(1,38)=22.2 ; p<0.0001)$, more distracted by internal thought $(F(1,38)=30.44 ; p<0.0001)$ and less distracted by external stimuli $(F(1,38)=8.46 ; p=0.01)$, engaged more in rumination and worry $(F(1,38)=82.02 ; p<0.0001)$, and had an overall increase in reaction times $(F(1,38)=21.14 ; p<0.0001)$ suggesting difficulties in disengaging thoughts. The task was too easy to allow for accuracy analysis. Particularly, GAD patients had a stronger loss of attentional focus compared to controls as shown by the Induction $\times$ Group interaction $(F(1,38)=6.32 ; p<0.0001)$ and significant post hoc comparisons $(t(18)=5.60 ; p<0.0001)$. Moreover, GAD patients had an overall lower HRV then healthy participants, as indicated by the main effect of Group in this analysis $(F(1,38)=11.43 ; p=0.002)$.

\subsection{HRV as a marker for cognitive rigidity}

Significant correlations were found between baseline (preinduction) $\mathrm{HRV}\left(61.4 \pm 38.9 \mathrm{~ms}^{2}\right)$ and pre- to post-induction change scores in levels of perseverative cognition (assessed by the VAS "ruminating/worrying?"; $\Delta=40.3 \pm 38.5$ ), suggesting a higher increase in perseverative cognition in participants with lower HRV $(\mathrm{r}=-0.33 ; \mathrm{p}=0.04)$.

\subsection{Neurobiological substrates of the transition from spontaneous self-generated thoughts to attentional control}

The detection of infrequent targets deactivated brain regions primarily attributed to the default mode network. Across
Table 2

Maxima of regions showing a significant $(z>2.3$, cluster corrected at $\mathrm{p}<0.05)$ main effect of Induction (before vs after) across groups.

\begin{tabular}{lrllll}
\hline $\mathrm{Z}$ & \multicolumn{1}{c}{$\mathrm{x}$} & $\mathrm{y}$ & $\mathrm{z}$ & Hemisphere & Region of activation \\
\hline 4.22 & 12 & 56 & 22 & Right & Frontal Pole \\
3.56 & -14 & 56 & 26 & Left & Frontal Pole \\
3.55 & 8 & 42 & 38 & Right & Superior Frontal Gyrus \\
3.51 & 8 & 44 & 34 & Right & Superior Frontal Gyrus \\
3.06 & 18 & 28 & 34 & Right & Superior Frontal Gyrus \\
3 & 18 & 18 & 46 & Right & Superior Frontal Gyrus \\
\hline
\end{tabular}

groups, discounting induction effects, target presentation elicited decreases in activation ( Pre $_{\text {tgt }}>$ Post $_{\text {tgt }}$ ) in regions of the left precuneus, postcentral gyrus and Heschl's Gyrus, and, bilaterally, regions of the precentral gyrus, juxtapositional lobule cortex, lateral occipital cortex, and occipital pole (Table 1a, Fig. 1A1). Increased activation following target presentation (Post $_{\text {tgt }}>$ Pre $_{\text {tgt }}$ ) was noted in Crus I and Crus II of the left cerebellum (Table 1b, Fig. 1A2).

\subsection{Neurobiological substrates of abnormal inhibitory processes following the induction}

A significant main effect of Induction and a Group $\times$ Induction interaction were shown: Both occurred for the Pre tgt $>$ Post $_{\text {tgt }}$ contrast. No supratheshold main effect of Group was observed.

The induction altered neural responses from pre- to post-target appearance $\left(\right.$ Pre $_{\text {tgt }}>$ Post $_{\text {tgt }}$ ) in areas of the frontal pole (bilaterally), superior frontal gyrus (right), and paracingulate gyrus (right) (see Table 2, Fig. 1B). Examination of the coefficient of parameter estimate (COPE) values from a regional mask encompassing these regions showed that target appearance evoked a greater reduction in activation post induction, compared to pre induction (Fig. 1B1).

A Group $\times$ Induction interaction ( Pre $_{\text {tgt }}>$ Post $_{\text {tgt }}$ ) was evident within precuneus (bilateral), lateral occipital gyrus (right), and posterior cingulate gyrus (bilateral) (see Table 3). Examination of the COPE estimates for a regional mask based on this interaction indicated that, after the induction, the activation patterns of the two groups tended to converge. The significant interaction was driven by a stronger reduction of activation in healthy individuals after 

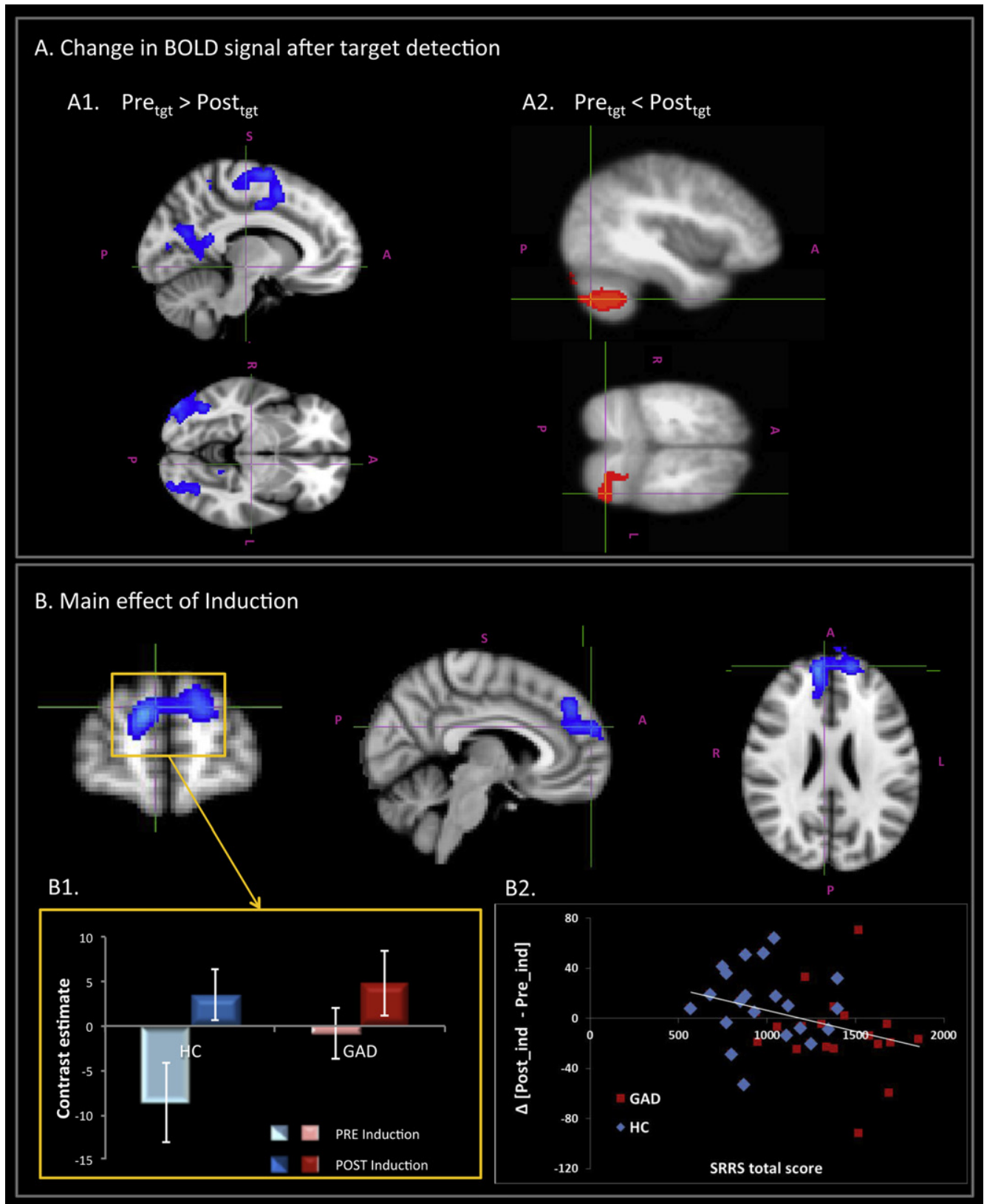

\section{Group x Induction interaction}
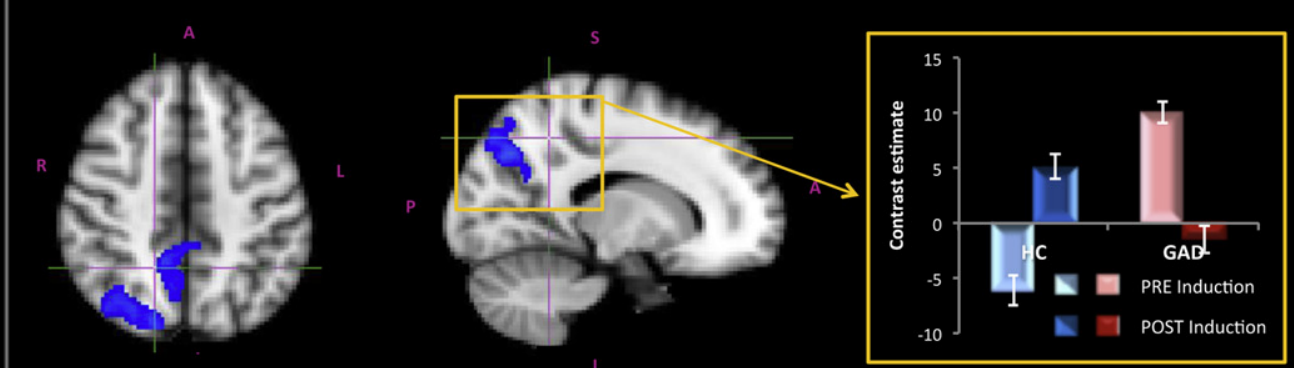

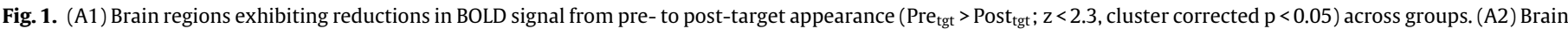

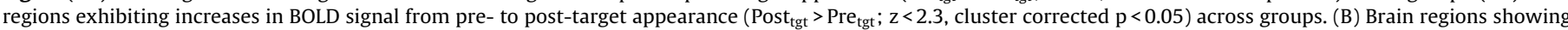

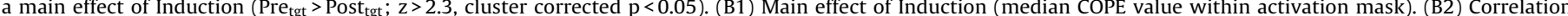

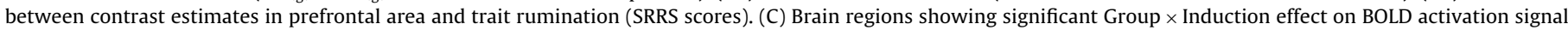

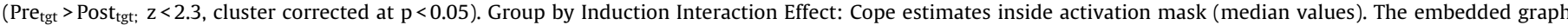
shows the Group by Induction Interaction Effect (median COPE value within activation mask). 
Table 3

Maxima of regions showing a significant $(z>2.3$, cluster corrected at $\mathrm{p}<0.05)$ Group (GAD vs HC) by Induction (before vs after) interaction.

\begin{tabular}{llllll}
\hline $\mathrm{Z}$ & $\mathrm{x}$ & $\mathrm{y}$ & $\mathrm{z}$ & Hemisphere & Region of activation \\
\hline 3.71 & 30 & -72 & 52 & Right & Lateral Occipital Gyrus \\
3.65 & -6 & -54 & 60 & Left & Precuneus \\
3.36 & 36 & -66 & 52 & Right & Lateral Occipital Gyrus \\
3.33 & 12 & -74 & 36 & Right & Precuneus \\
3.3 & 16 & -70 & 38 & Right & Precuneus \\
3.22 & 36 & -62 & 54 & Right & Lateral Occipital Gyrus \\
\hline
\end{tabular}

target presentation from pre- to post-induction while, in the GAD group, this reduction was already evident before induction (Fig. 1C).

\subsection{Additional analysis of target occurrence}

To confirm that the reported findings reflected the properties of transition between attentional states rather than target detection, a follow-up event-related analysis using the target presentation times as the events and event length as length of target display (i.e., $500 \mathrm{~ms}$ ) was conducted. Pre-induction, there was a main effect of group, the HC group displayed greater activation than GAD in a cluster located in the right frontal pole $\left(z_{\max }=3.96\right.$, MNI coordinates 26,56,6; $z>2.3$, cluster corrected $p<0.05$; online Supplement Table 1). This effect was absent in the post-induction analysis. Neither a main effect of Induction nor a Group $\mathrm{x}$ Induction interaction emerged from this analysis further supporting the view that the above results do not reflect target detection per se (online Supplement Fig. 1).

\subsection{Individual differences in trait perseverative cognition and inhibitory processes}

Correlational analyses were undertaken to examine relationships between neural responses from pre- to post-target appearance and SRRS. Both contrasts (Pre tgt $>$ Post $_{\text {tgt }}$ and Post $_{\text {tgt }}>$ Pre $_{\text {tgt }}$ ) were tested. In both groups, scores on the SRRS were significantly associated with the shift in activation following the induction (Fig. 1B2). More precisely, after the induction participants with higher dispositional tendencies to engage in perseverative cognition showed less deactivation within frontal pole regions at target presentation compared to low-trait SRRS individuals suggesting difficulties in disengaging thoughts.

\subsection{Individual differences in HRV and inhibitory processes}

Individual differences in HRV predicted the capacity for neural activity to shift from an internally-directed pattern supporting perseverative negative thoughts, to activity associated with control of externally-directed attention. HRV correlated negatively with both pre- and post-induction decreases in neural responses evoked by target presentation ( Pre $_{\text {tgt }}>$ Post $_{\text {tgt }}$ ). In other words, as HRV decreased, the magnitude of activation changes brought about by target detection increased. Before induction, HRV correlated with target-induced deactivation within right superior parietal lobule, supramarginal gyrus and superior frontal gyrus (Table 4a, Fig. 2a). Following the induction, HRV negatively correlated with targetinduced decreased activity bilaterally within central and parietal opercular cortices and precentral and postcentral gyri (Table $4 \mathrm{~b}$, Fig. 2b).

HRV did not correlate with target-related changes in activity before induction. However, following the induction, a negative correlation was noted for regions that encompassed right middle and inferior temporal gyri and Crus I and Crus II regions of the cerebellum: as HRV increased, there was less activation in these regions from pre- to post-target appearance (Fig. 2c).

\section{Discussion}

Combining neuroimaging techniques with peripheral physiology monitoring provides insight into brain-body interactions and the integration of autonomic, affective, and cognitive processes (Critchley, 2009). To our knowledge, this is the first functional imaging study in which simultaneous neuroimaging and autonomic recording were used to investigate difficulties in inhibiting perseverative thoughts and autonomic arousal, the most pervasive symptoms of GAD. We first examined the brain areas associated with the transition from spontaneous self-generated thoughts to attentional control during a low-demanding task in GAD and healthy participants. Second, we studied the consequences of a perseverative cognition induction on this shift from internally- to externally-oriented attention. Then, we tested if the effects of the induction on this attentional shift could be predicted by dispositional tendencies to engage in perseverative cognition (scores on the SRRS). Lastly, we examined the association between HRV and brain activation changes during the transition from internal thoughts to task focusing.

The neural responses elicited by target appearance were principally expressed as a relative deactivation rather than activation within specific brain regions. This was particularly the case for regions which form part of the default mode network, including the precuneus, which is implicated in episodic memory, visuospatial processing, self-reflection and aspects of consciousness (Fox, Spreng, Ellamil, Andrews-Hanna, \& Christoff, 2015; Hannawi, Lindquist, Caffo, Sair, \& Stevens, 2015; Kwok \& Macaluso, 2015). Target appearance also attenuated activity within motor, auditory, and somatosensory areas, putatively reflecting the rich multisensory nature of internal thoughts (e.g., Fox, Nijeboer, Solomonova, Domhoff, \& Christoff, 2013; Hasenkamp et al., 2012). Perhaps surprisingly, we did not find target-related changes within default mode network regions, notably posterior cingulate cortex, which is also linked to self-referential thoughts and worry (e.g., Christoff, Gordon, Smallwood, Smith, \& Schooler, 2009; Paulesu et al., 2010). Present results are, however, consistent with a recent metaanalysis of neuroimaging studies, which concluded that default mode activation alone is insufficient to capture fully the neural basis of spontaneous thought (Fox et al., 2015). Also, in the present study we sought to address the concept of cognitive "flexibility" by specifically targeting transitions from internal thoughts to externally oriented attention, and therefore focused on a different research question compared to studies directly comparing brain correlates of worrisome and neutral thoughts (e.g., Hoehn-Saric et al., 2004; Paulesu et al., 2010).

We replicated the effects of a perseverative cognition induction (e.g., Ottaviani \& Shapiro, 2011; Ottaviani, Shapiro, \& Fitzgerald, 2011) on target detection performance, showing decreased focus on the task and slower reaction times to target appearance indicative of difficulties in getting out of a state of heighted focus on ruminative and worrisome thoughts (Franklin, Smallwood, \& Schooler, 2011; Ottaviani et al., 2013). Moreover, baseline HRV was associated with pre-to post-induction changes in perseverative cognition, confirming the previously reported association between cognitive inflexibility and autonomic rigidity (Ottaviani et al., 2013; Ottaviani, Medea et al., 2015; Ottaviani, Shahabi et al., 2015).

In line with the notion that worry in GAD is likely to be more quantitatively than qualitatively different from normal worry (Borkovec, Alcaine, \& Behar, 2004; Ruscio \& Borkovec, 2004), the induction increased the amount of perseverative negative thoughts in controls up to an equivalent level reported by GAD patients, making the two groups more similar.

Our implementation of an induction procedure extended what has been previously observed on changes in brain activity: an earlier study reported that worry-inducing sentences, enhances 
Table 4

Maxima of regions showing a significant negative correlation ( Pre $_{\text {tgt }}>$ Post $_{\text {tgt }}$ ) with $\ln (\mathrm{HRV})$ pre and post induction.

\begin{tabular}{|c|c|c|c|c|c|c|}
\hline Contrast & Z & $\mathrm{x}$ & $\mathrm{y}$ & $\mathrm{z}$ & Hemisphere & Region of activation \\
\hline \multirow[t]{6}{*}{ (a) Pre $_{\text {tgt }}>$ Post $_{\text {tgt }}$ pre-induction } & 3.89 & 44 & -46 & 62 & Right & Superior Parietal Lobule \\
\hline & 3.74 & 38 & -46 & 50 & Right & Superior Parietal Lobule \\
\hline & 3.72 & 34 & -10 & 38 & Right & Precentral Gyrus \\
\hline & 3.22 & 56 & -34 & 60 & Right & Supramarginal Gyrus \\
\hline & 3.18 & 18 & 2 & 60 & Right & Superior Frontal Gyrus \\
\hline & 3.11 & 52 & -34 & 58 & Right & Supramarginal Gyrus \\
\hline \multirow[t]{12}{*}{ (b) Pre $_{\text {tgt }}>$ Post $_{\text {tgt }}$ post-induction } & 4.41 & -58 & -6 & 6 & Left & Central Opercular Cortex \\
\hline & 4.34 & -52 & -8 & 8 & Left & Central Opercular Cortex \\
\hline & 4.27 & -50 & -6 & 42 & Left & Precentral Gyrus \\
\hline & 4.12 & -38 & -20 & 50 & Left & Precentral Gyrus \\
\hline & 3.49 & -60 & -8 & 16 & Left & Postcentral Gyrus \\
\hline & 3.46 & -42 & -14 & 30 & Left & Postcentral Gyrus \\
\hline & 5.06 & 62 & 0 & 8 & Right & Central Opercular Cortex \\
\hline & 4.62 & 56 & -6 & 26 & Right & Postcentral Gyrus \\
\hline & 4.08 & 56 & -32 & 24 & Right & Parietal Opercular Cortex \\
\hline & 3.62 & 64 & -16 & 20 & Right & Postcentral Gyrus \\
\hline & 3.57 & 58 & -2 & 40 & Right & Precentral Gyrus \\
\hline & 3.55 & 72 & -18 & 16 & Right & Postcentral Gyrus \\
\hline
\end{tabular}

the activity within medial prefrontal cortices when compared to neutral sentences in both GAD patients and controls (Paulesu et al., 2010). We observed an increase in activity after induction within frontal pole, superior frontal gyrus, and paracingulate gyrus, regions implicated in self-referential processing and introspection. These regions decreased their responses to target appearance suggesting a role in self-related mental imagery. Induction amplified the pre-target engagement of these regions in perseverative cognition, particularly in healthy controls since it is likely that in GAD patients this effect was already present before the induction. Thus, the induction had the effect to make the two groups more similar both at a behavioural and at a neural level. These findings extend to anxiety disorder previous observations from pathological worriers (Ottaviani et al., 2014) and patients with major depression (Ottaviani, Shahabi et al., 2015).

A dimensional approach to psychopathological symptoms was further supported by the finding that scores on a continuous measure of dispositional perseverative cognition (SRRS) also predicted these effects of induction on prefrontal areas activation. High trait participants were characterized by less prefrontal deactivation at target appearance after the induction compared to low trait participants. Though focusing on worry, a similar relationship has been found between scores on the PSWQ and sustained activation of the medial frontal region after worry-induction (Paulesu et al., 2010). Together, these findings converge to suggest that these brain areas mediate the link between perseverative cognition and both the anticipation of threat and the lack of recovery from threat.

Participants with lower pre-induction HRV were characterized by stronger activation changes in areas such as the superior parietal lobule, supramarginal gyrus, and superior frontal gyrus, all critically important for the manipulation of information in working memory (du Boisgueheneuc et al., 2006; Koenigs, Barbey, Postle, \& Grafman, 2009), spontaneous thought processes (Fox et al., 2015), and the dynamic switch between external and internal modes of attention (Andrews-Hanna et al., 2014). This is a counterintuitive result, as one may expect higher (and not lower) HRV to index a better ability to switch from internally-oriented to externally-oriented attention, and hence be associated with stronger deactivation in such areas. Nevertheless, participants with lower HRV (GAD patients in our sample) are likely to engage in more perseverative cognition compared to those with higher HRV. Thus, larger changes (i.e., stronger deactivation of these areas at target appearance) would be entirely due to baseline differences. This is supported by the significant correlation between baseline HRV and pre-to post-induction changes in perseverative cognition, consistent with established relationships between HRV and performance on cognitive tasks under threat conditions (Hansen, Johnsen, \& Thayer, 2003; Hansen, Johnsen, \& Thayer, 2009).

Interestingly, pre- and post-induction associations implicated different regions, suggesting that HRV is able to predict differences in brain activity during both "normal" mind wandering and dysfunctional perseverative cognition. Whereas before the induction, HRV was associated with activity principally within working memory areas, after the induction lower levels of HRV were correlated with a stronger deactivation of somatosensory areas (postcentral gyrus, parietal operculum), reflecting the idea that perseverative cognition is akin to being more immersed in imagined situations. Future fMRI studies directly comparing functional and dysfunctional forms of repetitive thinking are needed to clarify if mind wandering and perseverative cognition can be differentiated by the degree to which they recruit attentional or salience-related brain networks, respectively.

When we looked at post-induction activation patterns, participants with lower HRV levels expressed higher activation within the cerebellum and the inferior temporal gyri. The cerebellum is certainly involved in physiological regulation as a component of the central autonomic network (Spyer, 1999) contributing to the control of cardiovascular responses through its connections with the hypothalamus (Critchley, Corfield, Chandler, Mathias, \& Dolan, 2000). Accordingly, covariation is observed between HRV and the cerebellum (Critchley et al., 2003; Gianaros, Van Der Veen, \& Jennings, 2004; Lane et al., 2009; Napadow et al., 2008).

The temporal lobe regions are associated with counterfactual reasoning (Van Hoeck et al., 2014), including retrieving, reliving and elaborating past events (conscious episodic memory al (Addis, Wong, \& Schacter, 2007; Andreasen et al., 1999) and imagining potential futures (Summerfield, Hassabis, \& Maguire, 2010; Weiler, Suchan, \& Daum, 2010). Our data raise the intriguing notion that there may be physiological constraints on counterfactual thought. Low tonic levels of HRV might indicate a predisposition to keep on 'erring on the side of caution' (Gerin et al., 2012) reflected in perseverative thinking on the psychological level. Here, we provided the first evidence for brain areas mediating this relationship by bridging the central autonomic and the cingulo-opercular networks.

Our findings add to knowledge regarding HRV as a potential marker of stress and health (Thayer et al., 2012), shedding light into the still unexplained relationship between anxiety disorders and cardiovascular risk (see Player \& Peterson, 2011 for a review). Considering that perseverative cognition is one of the most difficult symptoms to treat in anxiety, our results may also have important 

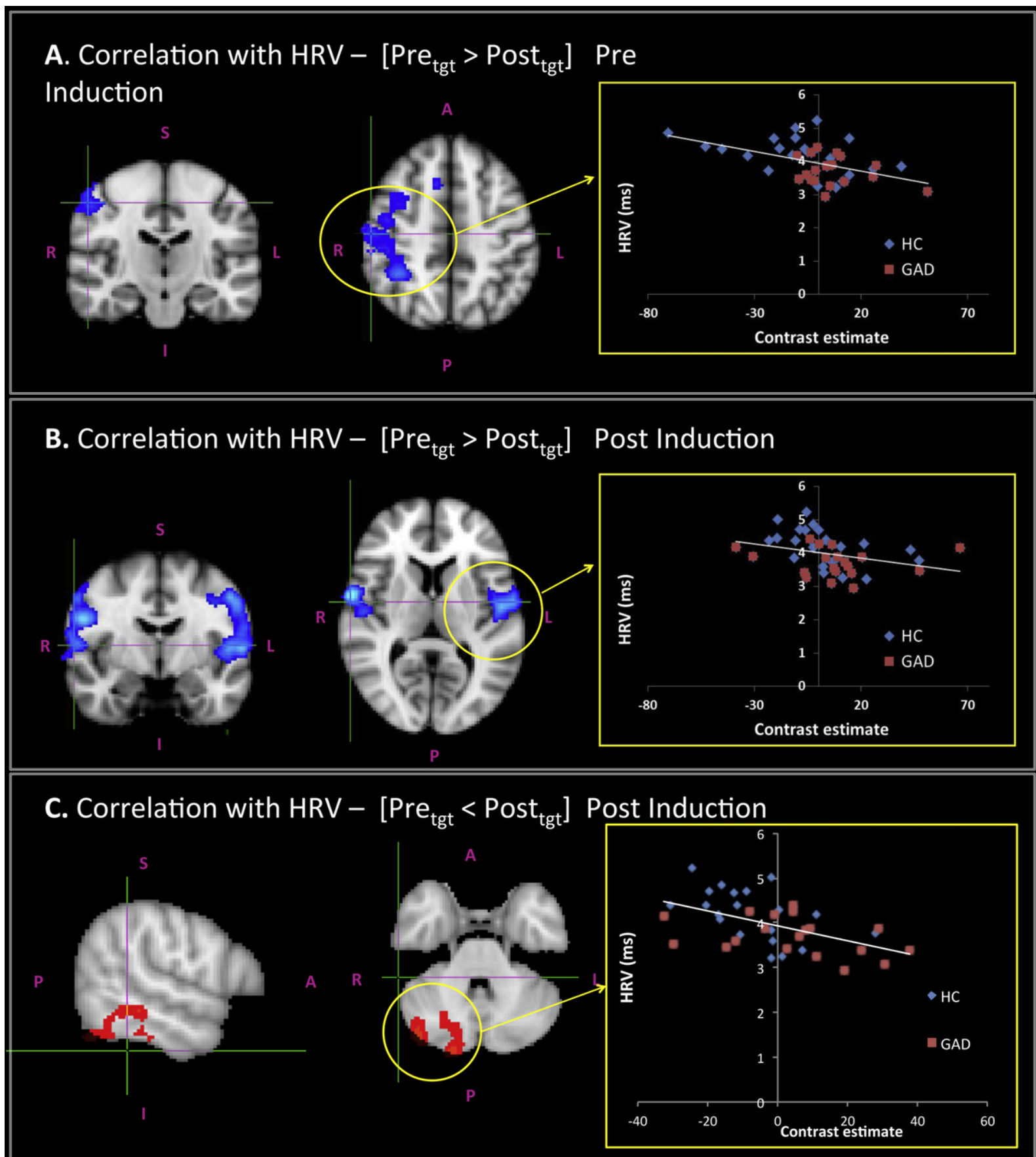

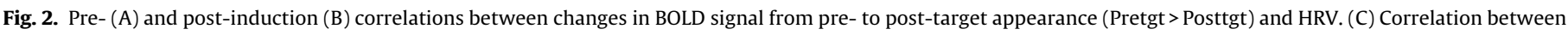

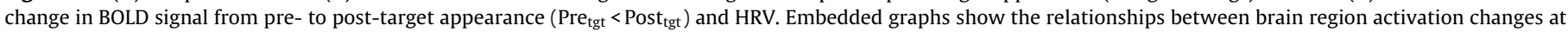
target appearance (median COPE value within activation mask) and $\ln (\mathrm{HRV})$.

implications for therapy. HRV is a significant predictor of treatment response and remission from major depression (Chambers \& Allen, 2002; Jain et al., 2014), and HRV biofeedback is a promising tool for the treatment of mood disorder (Karavidas et al., 2007). Our results argue for the extension of these pilot intervention studies to anxiety disorders. Moreover, in a research domain, our results suggest HRV is a useful tool to detect and index perseverative cognition without interrupting or interfering with free thinking. Given that spontaneity is a key feature of intrusive thoughts, future investigations may increase ecological validity by triggering thought probes based on the physiological (i.e., HRV) changes of study participants.
We acknowledge that the statistical power of our study will likely improve with a larger sample size. A second limitation is that the occurrence of perseverative cognition while participants are not focusing on a task could only be assumed. To reduce this bias, we chose an induction manipulation that has been extensively proven to be effective in eliciting worrisome and ruminative thoughts. Moreover, an increase in these types of thoughts from pre- to postinduction was testified by self-report measures (VAS). Limitations notwithstanding, our findings highlight the dynamic relationship between mind and body by identifying neural substrates that shape aberrant cognitions in anxiety through psychophysiological interaction with autonomic bodily state. An integrative investigative 
approach is called for to better inform therapeutic interventions, combining techniques including functional brain imaging and detailed autonomic monitoring, and drawing on both basic research and clinical observations.

\section{Financial disclosures}

The authors report no biomedical financial interests or potential conflicts of interest.

\section{Acknowledgments}

This work was supported by the Italian Ministry of Health Young Researcher Grant awarded to Dr Cristina Ottaviani (GR2010-2312442).

\section{Appendix A. Supplementary data}

Supplementary data associated with this article can be found, in the online version, at http://dx.doi.org/10.1016/j.biopsycho.2016. 06.009.

\section{References}

Addis, D. R., Wong, A. T., \& Schacter, D. L. (2007). Remembering the past and imagining the future: common and distinct neural substrates during event construction and elaboration. Neuropsychologia, 45, 1363-1377.

Andreasen, N. C., O'Leary, D. S., Paradiso, S., Cizadlo, T., Arndt, S., Watkins, G. L., et al. (1999). The cerebellum plays a role in conscious episodic memory retrieval. Human Brain Mapping, 8, 226-234.

Andrews-Hanna, J. R., Smallwood, J., \& Spreng, R. N. (2014). The default network and self-generated thought: component processes, dynamic control, and clinical relevance. Annals of the New York Academy of Sciences, 1316, 29-52.

Anticevic, A., Cole, M. W., Murray, J. D., Corlett, P. R., Wang, X. J., \& Krystal, J. H. (2012). The role of default network deactivation in cognition and disease. Trends in Cognitive Science, 16, 584-592.

Beck, A. T., \& Steer, R. A. (1984). Internal consistencies of the original and revised Beck depression inventory. Journal of Clinical Psychology, 40, 1365-1367.

Beck, A. T., Steer, R. A., \& Garbin, M. G. J. (1988). Psychometric properties of the Beck depression inventory twenty-five years of evaluation. Clinical Psychological Review, 8, 77-100.

Beesdo, K., Pine, D. S., Lieb, R., \& Wittchen, H. U. (2010). Incidence and risk patterns of anxiety and depressive disorders and categorization of generalized anxiety disorder. Archives of General Psychiatry, 67, 47-57.

Binder, J. R., Frost, J. A., Hammeke, T. A., Bellgowan, P. S., Rao, S. M., \& Cox, R. W. (1999). Conceptual processing during the conscious resting state: a functional MRI study. Journal of Cognitive Neuroscience, 11, 80-95.

Borkovec, T. D., Alcaine, O., \& Behar, E. S. (2004). Avoidance theory of worry and generalized anxiety disorder. In R. Heimberg, D. Mennin, \& C. Turk (Eds.), Generalized anxiety disorder: advances in research and practice (pp. 77-108). New York: Guilford.

Brown, T. A., Antony, M. M., \& Barlow, D. H. (1992). Psychometric properties of the Penn State Worry Questionnaire in a clinical anxiety disorders sample. Behavior Research and Therapy, 30, 33-37.

Chambers, A. S., \& Allen, J. J. (2002). Vagal tone as an indicator of treatment response in major depression. Psychophysiology, 39, 861-864.

Christoff, K., Gordon, A. M., Smallwood, J., Smith, R., \& Schooler, J. W. (2009). Experience sampling during fMRI reveals default network and executive system contributions to mind wandering. Proceedings of the National Academy of Sciences of the United States of America, 106, 8719-8724.

Critchley, H. D. (2009). Psychophysiology of neural, cognitive and affective integration: fMRI and autonomic indicants. International Journal of Psychophysiology, 73, 88-94.

Critchley, H. D., Corfield, D. R., Chandler, M. P., Mathias, C. J., \& Dolan, R. J. (2000). Cerebral correlates of autonomic cardiovascular arousal: a functional neuroimaging investigation in humans. Journal of Physiology, 523, 259-270.

Critchley, H. D., Eccles, J., \& Garfinkel, S. N. (2013). Interaction between cognition, emotion, and the autonomic nervous system. Handbook of Clinical Neurology, 117, 59-77.

Critchley, H. D., Mathias, C. J., \& Dolan, R. J. (2002). Fear conditioning in humans: the influence of awareness and autonomic arousal on functional neuroanatomy. Neuron, 33, 653-663.

Critchley, H. D., Mathias, C. J., Josephs, O., O’Doherty, J., Zanini, S., Dewar, B. K., et al. (2003). Human cingulate cortex and autonomic control: converging neuroimaging and clinical evidence. Brain, 126, 2139-2152.

du Boisgueheneuc, F., Levy, R., Volle, E., Seassau, M., Duffau, H., Kinkingnehun, S., et al. (2006). Functions of the left superior frontal gyrus in humans: a lesion study. Brain, 129, 3315-3328.
Forster, S., Nunez Elizalde, A. O., Castle, E., \& Bishop, S. J. (2015). Unraveling the anxious mind: anxiety, worry, and frontal engagement in sustained attention versus off-task processing. Cerebral Cortex, 25, 609-618.

Fox, K. C., Nijeboer, S., Solomonova, E., Domhoff, G. W., \& Christoff, K. (2013). Dreaming as mind wandering: evidence from functional neuroimaging and first-person content reports. Frontiers in Human Neuroscience, 7, 412. http://dx. doi.org/10.3389/fnhum.2013.00412

Fox, K. C., Spreng, R. N., Ellamil, M., Andrews-Hanna, J. R., \& Christoff, K. (2015). The wandering brain: meta-analysis of functional neuroimaging studies of mind-wandering and related spontaneous thought processes. NeuroImage, 11, 611-621.

Franklin, M. S., Smallwood, J., \& Schooler, J. W. (2011). Catching the mind in flight: using behavioral indices to detect mindless reading in real time. Psychonomic Bulletin E Review, 18, 992-997.

Friedman, B. H. (2007). An autonomic flexibility-neurovisceral integration model of anxiety and cardiac vagal tone. Biological Psychology, 74, 185-199.

Friedman, B. H., \& Thayer, J. F. (1998). Autonomic balance revisited: panic anxiety and heart rate variability. Journal of Psychosomatic Research, 44, 133-151.

Garfinkel, S. N., \& Critchley, H. D. (2016). Threat and the body: how the heart supports fear processing. Trends in Cognitive Science, 20, 34-46.

Gerin, W., Zawadzki, M. J., Brosschot, J. F., Thayer, J. F., Christenfeld, N. J., Campbell, T. S., et al. (2012). Rumination as a mediator of chronic stress effects on hypertension: a causal model. International Journal of Hypertension, 453465. http://dx.doi.org/10.1155/2012/453465

Gianaros, P. J., Van Der Veen, F. M., \& Jennings, J. R. (2004). Regional cerebral blood flow correlates with heart period and high-frequency heart period variability during working-memory tasks: implications for the cortical and subcortical regulation of cardiac autonomic activity. Psychophysiology, 41, 521-530.

Hannawi, Y., Lindquist, M. A., Caffo, B. S., Sair, H. I., \& Stevens, R. D. (2015). Resting brain activity in disorders of consciousness: a systematic review and meta-analysis. Neurology, 84, 1272-1280.

Hansen, A. L., Johnsen, B. H., \& Thayer, J. F. (2003). Vagal influence on working memory and attention. International Journal of Psychophysiology, 48, 263-274.

Hansen, A. L., Johnsen, B. H., \& Thayer, J. F. (2009). Relationship between heart rate variability and cognitive function during threat of shock. Anxiety, Stress, and Coping, 22, 77-89.

Hasenkamp, W., Wilson-Mendenhall, C. D., Duncan, E., Barsalou, L. W., Hoehn-Saric, R., \& Schlund, M. W. (2012). Mind wandering and attention during focused meditation: a fine-grained temporal analysis of fluctuating cognitive states. Neurolmage, 59, 750-760.

He, J., Becic, E., Lee, Y. C., \& McCarley, J. S. (2011). Mind wandering behind the wheel: performance and oculomotor correlates. Human Factors, 53, 13-21.

Hoehn-Saric, R., Schlund, M. W., \& Wong, S. H. (2004). Effects of citalopram on worry and brain activation in patients with generalized anxiety disorder. Psychiatry Research, 131, 11-21.

Jain, F. A., Cook, I. A., Leuchter, A. F., Hunter, A. M., Tartter, M., Davydov, D. M., et al. (2014). Heart rate variability and treatment outcome in major depression: a pilot study. International Journal of Psychophysiology, 93, 204-210.

Jenkinson, M., Bannister, P., Brady, M., \& Smith, S. (2002). Improved optimization for the robust and accurate linear registration and motion correction of brain images. NeuroImage, 17, 825-841.

Jenkinson, M., \& Smith, S. (2001). A global optimisation method for robust affine registration of brain images. Medical Image Analysis, 5, 143-156.

Karavidas, M. K., Lehrer, P. M., Vaschillo, E., Vaschillo, B., Marin, H., Buyske, S., et al. (2007). Preliminary results of an open label study of heart rate variability biofeedback for the treatment of major depression. Applied Psychophysiology and Biofeedback, 32, 19-30.

Klinger, E. (1978). Modes of normal conscious flow. In K. S. Pope, \& J. L. Singer (Eds.), The stream of consciousness: scientific investigations into the flow of human experience (pp. 225-258). New York: Plenum.

Koenigs, M., Barbey, A. K., Postle, B. R., \& Grafman, J. (2009). Superior parietal cortex is critical for the manipulation of information in working memory. Journal of Neuroscience, 29, 14980-14986.

Kwok, S. C., \& Macaluso, E. (2015). Immediate memory for when, where and what: short-delay retrieval using dynamic naturalistic material. Human Brain Mapping, 36, 2495-2513.

Lane, R. D., McRae, K., Reiman, E. M., Chen, K., Ahern, G. L., \& Thayer, J. F. (2009). Neural correlates of heart rate variability during emotion. NeuroImage, 44 , 213-222.

Makovac, E., Meeten, F., Watson, D. R., Garfinkel, S. N., Critchley, H. D., \& Ottaviani, C. (2015). Neurostructural abnormalities associated with axes of emotion dysregulation in generalized anxiety. NeuroImage: Clinical, 10, 172-181.

Mason, M. F., Norton, M. I., Van Horn, J. D., Wegner, D. M., Grafton, S. T., \& Macrae, C. N. (2007). Wandering minds: the default network and stimulus-independent thought. Science, 315, 393-395.

McEvoy, P. M., Watson, H., Watkins, E. R., \& Nathan, P. (2013). The relationship between worry, rumination, and comorbidity: evidence for repetitive negative thinking as a transdiagnostic construct. Journal of Affective Disorders, 151, 313-320.

Meyer, T. J., Miller, M. L., Metzger, R. L., \& Borkovec, T. D. (1990). Development and validation of the penn state worry questionnaire. Behavior Reseach and Therapy, 28, 487-495.

Molina, S., \& Borkovec, T. D. (1994). The penn state worry questionnaire: psychometric properties and associated characteristics. In G. C. L. Davey, \& F. Tallis (Eds.), Worrying: perspectives on theory, assessment, and treatment ( $\mathrm{pp}$. 265-283). New York: Wiley. 
Mullinger, K. J., Mayhew, S. D., Bagshaw, A. P., Bowtell, R., \& Francis, S. T. (2013). Poststimulus undershoots in cerebral blood flow and BOLD fMRI responses are modulated by poststimulus neuronal activity. Proceedings of the National Academy of Sciences of the United States of America, 110, 13636-13641.

Napadow, V., Dhond, R., Conti, G., Makris, N., Brown, E. N., \& Barbieri, R. (2008). Brain correlates of autonomic modulation: combining heart rate variability with fMRI. NeuroImage, 42, 169-177.

Northoff, G., Qin, P., \& Nakao, T. (2010). Rest-stimulus interaction in the brain: a review. Trends in Neuroscience, 33, 277-284

Nussinovitch, U., Elishkevitz, K. P., Katz, K., Nussinovitch, M., Segev, S., Volovitz, B., et al. (2011). Reliability of ultra-short ECG indices for heart rate variability. Annals of Noninvasive Electrocardiology, 16, 117-122.

Ottaviani, C., Borlimi, R., Brighetti, G., Caselli, G., Favaretto, E., Giardini, I., et al. (2014). Worry as an adaptive avoidance strategy in healthy controls but not in pathological worriers. International Journal of Psychophysiology, 93, 349-355.

Ottaviani, C., Medea, B., Lonigro, A., Tarvainen, M., \& Couyoumdjian, A. (2015). Cognitive rigidity is mirrored by autonomic inflexibility in daily life perseverative cognition. Biological Psychology, 107, 24-30.

Ottaviani, C., Shahabi, L., Tarvainen, M., Cook, I., Abrams, M., \& Shapiro, D. (2015). Cognitive, behavioral, and autonomic correlates of mind wandering and perseverative cognition in major depression. Frontiers in Neuroscience, 8, 433.

Ottaviani, C., \& Shapiro, D. (2011). Do we need a stressor to be stressed? Insights from cardiac regulation. Japanese Psychological Research, 53, 155-162.

Ottaviani, C., Shapiro, D., \& Couyoumdjian, A. (2013). Flexibility as the key for somatic health: from mind wandering to perseverative cognition. Biological Psychology, 94, 38-43.

Ottaviani, C., Shapiro, D., \& Fitzgerald, L. (2011). Rumination in the lab: what happens when you go back to everyday life? Psychophysiology, 48, 453-461.

Ottaviani, C., Thayer, J. F., Verkuil, B., Lonigro, A., Medea, B., Couyoumdjian, A., et al. (2016). Physiological concomitants of perseverative cognition: a systematic review and meta-analysis. Psychological Bullettin, 142, 231-259.

Paulesu, E., Sambugaro, E., Torti, T., Danelli, L., Ferri, F., Scialfa, G., et al. (2010) Neural correlates of worry in generalized anxiety disorder and in normal controls: a functional MRI study. Psychological Medicine, 40, 117-124.

Player, M. S., \& Peterson, L. E. (2011). Anxiety disorders, hypertension, and cardiovascular risk: a review. International Journal of Psychiatry in Medicine, 41, 365-377.

Raichle, M. E., MacLeod, A. M., Snyder, A. Z., Powers, W. J., Gusnard, D. A., \& Shulman, G. L. (2001). A default mode of brain function. Proceedings of the National Academy of Sciences of the United States of America, 98, 676-682.
Robinson, M. S., \& Alloy, L. B. (2003). Negative cognitive styles and stress-reactive rumination interact to predict depression: a prospective study. Cognitive Therapy and Research, 27, 275-292.

Ruscio, A. M., \& Borkovec, T. D. (2004). Experience and appraisal of worry among high worriers with and without generalized anxiety disorder. Behavior Research and Therapy, 42, 1469-1482.

Segerstrom, S. C., Tsao, J. C. I., Alden, L. E., \& Craske, M. G. (2000). Worry and rumination: repetitive thought as a concomitant and predictor of negative mood. Cognitive Therapy and Research, 24, 671-688.

Smallwood, J. (2011). The footprints of a wandering mind: further examination of the time course of an attentional lapse. Cognitive Neuroscience, 2, 91-97.

Smallwood, J., Beach, E., Schooler, J. W., \& Handy, T. C. (2008). Going AWOL in the brain: mind wandering reduces cortical analysis of external events. Journal of Cognitive Neuroscience, 20, 458-469.

Spielberger, C. D., Gorsuch, R. L., Lushene, R., Vagg, P. R., \& Jacobs, G. A. (1983). Manual for the state-trait anxiety inventory. Palo Alto, CA: Consulting Psychologists Press.

Spinhoven, P., Drost, J., van Hemert, B., \& Penninx, B. W. (2015) Common rather than unique aspects of repetitive negative thinking are related to depressive and anxiety disorders and symptoms. Journal of Anxiety Disorder, 33, 45-52.

Spyer, K. M. (1999). Central nervous control of the cardiovascular system. In C. J. Mathias, \& R. Bannister (Eds.), Autonomic failure: a textbook of clinical disorders of the autonomic nervous system (pp. 45-55). Oxford, UK: Oxford University Press.

Summerfield, J. J., Hassabis, D., \& Maguire, E. A. (2010). Differential engagement of brain regions within a 'core' network during scene construction. Neuropsychologia, 48, 1501-1509.

Thayer, J. F., Ahs, F., Fredrikson, M., Sollers, J. J., 3rd, \& Wager, T. D. (2012). A meta-analysis of heart rate variability and neuroimaging studies: implications for heart rate variability as a marker of stress and health. Neuroscience and Biobehavioral Review, 36, 747-756.

Van Hoeck, N., Begtas, E., Steen, J., Kestemont, J., Vandekerckhove, M., \& Van Overwalle, F. (2014). False belief and counterfactual reasoning in a social environment. NeuroImage, 90, 315-325.

Watkins, E. D., Moulds, M., \& Mackintosh, B. (2005). Comparisons between rumination and worry in a non-clinical population. Behavior Research and Therapy, 43, 1577-1585.

Weiler, J. A., Suchan, B., \& Daum, I. (2010). When the future becomes the past: differences in brain activation patterns for episodic memory and episodic future thinking. Behavioural Brain Research, 212, 196-203. 